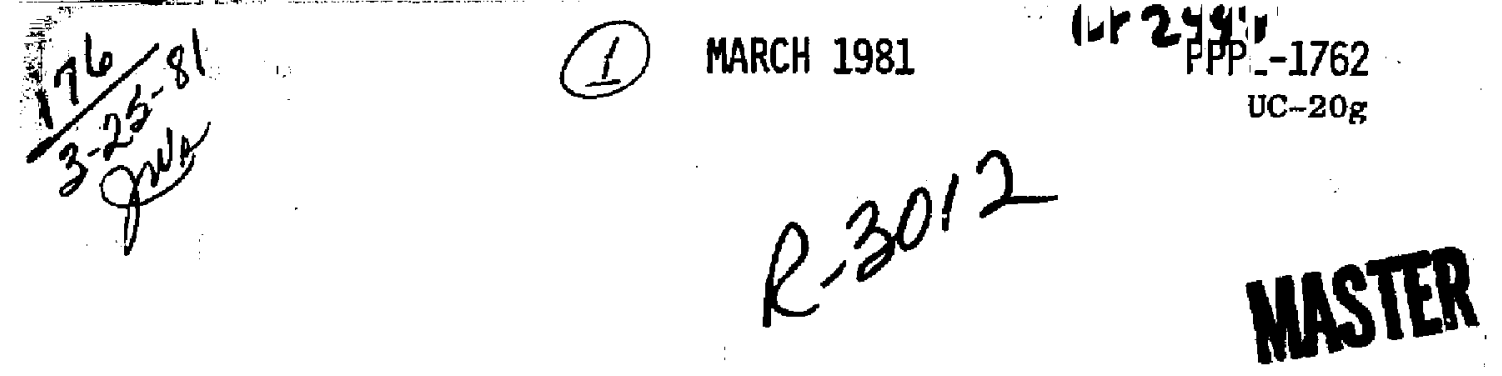

\title{
PLASMA EQUILIBRIUM WITH RATIONAL MAGNETIC SURFACES
}

BY

A, H, BOOZER

\section{PLASMA PHYSICS LABORATORY}

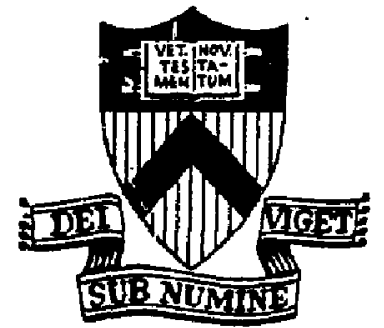

\section{PRINCETON \\ UNIVERSITY PRINCETON, NEW JERSEY}

This work supported by the $U$. S. Department of Energy Contract No. DE-AC02-76-CHO-3073. Reproduction, translation, publication, use and disposal, in whole or in part, by or for the United States Government is permitted. 


\title{
Plasma Equilibrium with Rational \\ Magnetic Surfaces
}

\author{
Allen H. Boozer \\ Princeton Plasma Physics Laboratory \\ Princeton Univergity \\ Princeton, New Jersey 08544
}

Mbstract

The self consigtent classical plasma equilibrium with diffuslon is studied in a toroidal geometry having a sheared magnetic field, Nar each rational surface 1 is found that the pressure gradient ts zero unless the Fourier component of $1 / \mathrm{B}^{2}$, which resonates with that surface, vantshes. Despite the resonances, the overall plasma confinement is, in practice, anly slightly modified by the rational surfaces.

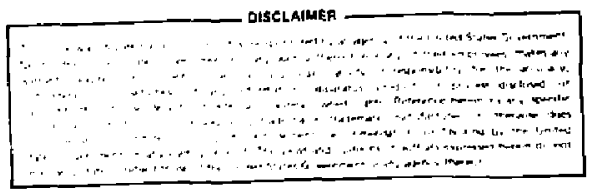




\section{Introduction}

Magnetic surfaces in which the rotation transform, $x$, is a rational number have long been known to be associated with singularities in the plasua equilibrium. An especially clear discussion of th1s problem has been given by H. Grad. 1 On surfaces with irrational $x$, the magnetic field lines ergodically cover the entire surfsce and the pressure is constant on the surface. On rational surfaces, the magnetic zield lines close on themselves. The condition for plasma equilibrlum with closed fleld lines is that the integral $f \mathrm{~d} / \mathrm{B}$ be constant on a pressure surface. In systems in which $x$ depends on radius, the pressure surfaces defingd by the trrational surfaces do not have $\int \mathrm{d} l / B$ constant on rational surfaces except for special cases such as toroidal symetry.

In this paper we will examine the problem of self-consistent plasma equilibrium in a toroidal system with shear. find that the plasma equilibrium is controlled by the fourier transform of $1 / \mathrm{B}^{2}$ in the appropriate torofdal and poloidal angles $\phi$ and $\theta$. Let $\delta_{n \pi}$ be proportional to the $n^{\text {th }}$ toroidal. harmonic and the $m^{\text {th }}$ poloidal harmonic of $1 / \mathrm{B}^{2}$, then near a rational gurface $x\left(\psi_{R}\right)=n / m$

$$
\begin{aligned}
& \left(j_{n}\right)_{P} \propto \frac{\delta_{n m}}{\left(\psi-\psi_{R}\right)} \frac{d P}{d \psi} \cos (n \phi-m \theta) \\
& I \propto-\eta_{1} \frac{\left|\delta_{n m}\right|^{2}}{\left(\psi-\psi_{R}\right)^{2}} \frac{d P}{d \psi} \\
& \phi-\phi_{0}(\psi) \propto \eta_{\|} \frac{\delta_{n m}}{\left(\psi-\psi_{R}\right)^{2}} \frac{d P}{d \psi} \sin (n \phi-m \theta)
\end{aligned}
$$


with ( $J_{\text {. }}$ ) the pressure driven part of the parallel current, $P$ the pressure, $\Gamma$

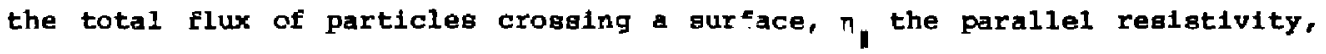
and $\$-\phi_{0}(\psi)$ the variation of the electrostatic potential in the surface. The smoothness of the particle flux implies $d P / d \psi \propto\left|\delta_{n m}\right|^{2}\left(\psi-\psi_{R}\right)^{2}$ near a rational ourface. Dnless $\left|\delta_{\mathrm{nm}}\right|$ vanishes, which is equivalent to $f \mathrm{~d} \ell / \mathrm{B}$ being constint on the rational surface, $d P / d \psi \times\left(\psi-\psi_{R}\right)^{2}$. Assuming $\left|\delta_{n m}\right| \neq 0$ we then find $\left(j_{N}\right) \propto\left(\psi-\psi_{R}\right)$, that is, it vanishes at $\psi=\psi_{R}$ while the potential variation remains finite. since $\left(j_{l_{P}}\right)$ vanishes everywhere as the plasma pressure goes to zero, any vacuum fleld configuration with magnetic surfaces and shear gives a plasma equilibrium at low encugh plasma pressure. Other papers which are related to the work reported heve are by Kruskal and Kulsrud, ${ }^{2}$ Hamada, ${ }^{3}$ Greene and Johnson, 4 and Grad. 5

In Section II of the paper the appropriate coordinate system will be established, in section III the equation for the parallel plasma current is derived and solved, in section IV the consequences of Ohm's law are explored, and the conclusions are given in section $v$.

\section{Magnetic Coordinates}

Solenolial vectors such as the magnetic field can always be written in the so-called clebsch representation

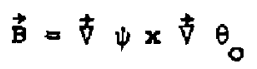

with a field line defined by constant $\psi$ and $\theta_{0}$. Since we are assuming a scaler preasure with 


$$
\text { 支 }=\frac{1}{c} \mathrm{~J} \times \text { 吉， }
$$

the clebsch coordinate $\psi$ can be chosen as a function of $P$ alone. The systems we are consldering have topologically toroldal pressure surfaces so the function $\psi(P)$ can be choosen with $2 \pi \psi$ equal to the magnetic flux inside a pressure surface (i.e., the torotalal flux). This choice of $\psi$ makes $\theta_{0}$ angle$1 \mathrm{~km} \cdot$

In addition to the clebsch or contravariant representation, a magnetic field with a scaler pressure can be written in the covariant furm ${ }^{6}$

$$
\vec{B}=\ddot{\nabla_{n}}+\beta \vec{\nabla} \psi
$$

with $\psi, \theta_{0}, x$ as coordinates. An important role is played by the arbitrariness in the $\psi, \theta_{0}, \chi, \beta$ representation of $\vec{B}$. Since $\psi$ is defined, this arbitraxiness occurs only in $\theta_{0}, x$, and $\beta$. It is easy to show that if $\theta_{0}, X$, and $B$ represent $\bar{B}$ then $\bar{\theta}_{0}, \bar{X}$, and $\bar{\beta}$ give a representation $i f$, and only if,

$$
\ddot{\theta}_{0}=\theta_{0}+\theta_{*}(\psi), \bar{x}=x+x_{*}(\omega), \bar{\beta}=\beta-\frac{d x_{*}}{d \psi} .
$$

The functions $\theta_{*}$ and $x_{*}$ are arbitrary functions of $\psi \cdot$

Although many fundomental properties of the plasma equations are easily 1lluatrated using $\theta_{0}$ and $x$ as coordinates, they do obscure the toroidal and the poloidal periodictties of the torus. Angular coordinates $q$ and $\$$ linearly related to $\theta_{i}$ and $x$ make this periodicity manicegt. Suppoee we circuit the torus once toroidally and come back to the same physical potnt in general $x$ 
and $\theta_{0}$ will not return to thelr original values $x(0)$ and $\theta_{0}(0)$. Rather after a torotdal circuit

$$
x=x(0)+2 \pi g, \theta_{0}=\theta_{0}(0)-2 \pi x .
$$

Both $x(0), \theta_{0}(0)$, and $x, \theta_{0}$ are representations of the field at the same physical location so $\mathrm{g}$ and $x$ must be functions of $\psi$ alone. In one polotal circuit

$$
x=x(0)+2 \pi I, \theta_{0}=\theta_{0}(0)+2 \pi \sigma
$$

Again $I$ and $\sigma$ must be functions of $\psi$ alone. The function $\sigma$ will be shown to equai the number one. The periodictties can be simply given by defining the poloidal angle $\theta$ and the toroidal angle $\phi$ so that

$$
\theta_{0}=\sigma \theta-x \phi \cdot x=9 \phi+I \theta \text {. }
$$

The coordinates of the paper will be $\psi, \theta, \phi$.

To show o is unity, remember that $2 \pi \psi$ equals the mangetic flux inslde a pressure surface or

$$
2 \pi \downarrow=\int t \cdot d E_{t} \cdot
$$

The element of surface area in $\psi, \theta$, coorlinates is

$$
d t_{t}=\frac{\nabla_{\phi}}{\nabla_{\phi} \cdot\left(\hat{\nabla}_{\psi \times} \hat{t}_{\theta}\right)} d \theta d \psi .
$$

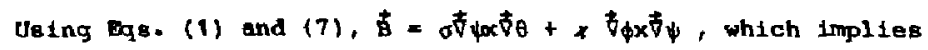




$$
\psi=\int \sigma d \psi .
$$

In $\psi, \theta, \phi$ coordinates the contravariant form of the magnetic field is then

$$
\vec{B}=\nabla_{\psi} \times \nabla_{\theta}+x(\phi) \nabla_{\phi} \times \vec{\nabla}_{\psi}
$$

while the covariant form is

$$
\vec{B}=g(\psi))_{\phi}+I\left(\psi^{\prime} \nabla_{\theta}+\beta_{\star} \vec{\gamma}_{\psi}, \beta_{\star}=\beta+\left(\frac{d g}{d \psi} \phi+\frac{d I}{d \psi} \theta\right) .\right.
$$

The total torofial current inside a flux surface is

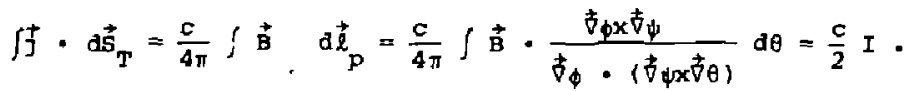

The total poloidal current outside a flux surface can similarly be shown to be $c g(\psi) / 2$.

\section{The Current Density}

The covartant representation of B, Bg. (13), gives a simple expression for the current denaity

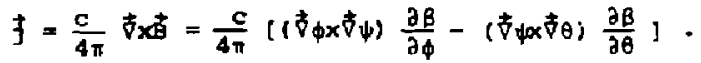

The cross product of this expression with the contravariant representation of B, Eq, $(12)$, gives an equation for $B$ 


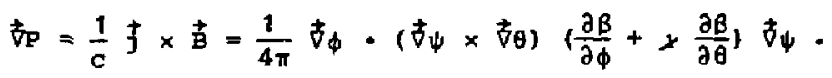

The inverse of the $\psi, \theta, \phi$ Jacobian can be found by dotting together the covariant, Eq. (13), and contravariant, Eq. (12), representations of

$$
\nabla_{\phi} \cdot(\vec{\nabla} \psi \times \text { 南 } \theta)=\frac{B^{2}}{9+x I} \cdot
$$

The equation for $\beta$ is then

$$
\frac{\partial B}{\partial \phi}+\chi \frac{\partial B}{\partial \theta}=\frac{4 n}{B^{2}}(g+x I) \frac{d P}{d \psi}
$$

The parallel component of $j$ can be found by dotting the covariant expression fox $\dot{B}$, Eq. (13), into $\}$, Eq. (15), to obtain

$$
\frac{4 \pi}{c} \frac{j}{\beta}-=\frac{1}{g+\angle I}\left(I \frac{\partial B}{\partial \phi}-q \frac{\partial B}{\partial \theta}\right) .
$$

Let us now solve the equations for $\beta$ and $j_{\|} / B$. The function $\beta$ need not be periodic in $\theta$ and $\phi$, however, $j_{n} / B$ must be. One finds a homogeneous solution for $B$.

$$
B_{H}=H_{H}(\psi)(x \phi-\theta)
$$

with $\mu_{H}$ an arbitrary function of $\psi$. To this solution the inhonogeneous solution to Eq. (18) must be added. To find the inhomogeneous solution let

$$
\frac{1}{B^{2}}=\frac{1}{B_{0}^{2}}\left[\left(1+\sum_{n, m}^{\prime} \delta_{n m} \cos \left(n \phi-m \theta+\lambda_{n m}\right)\right]\right.
$$


with the prime on the sum implying that the term $n=0, m=0$ ia eliminated. That is, we assume the field strength in each magnetic surface is known and that it can be approptiately expanded in a Fourler series. Then one finds

$$
B=H_{H}(\psi)(H \phi-\theta)+\frac{4 \pi}{B_{0}^{2}}(g+x I) \frac{d P}{d \psi} \phi+B
$$

with $\beta_{*}$, which we will presently show is the $B_{*}$ of Bq. (13), equal to

$$
B_{*}=\frac{4 \pi}{B_{0}^{2}} \frac{d P}{d \psi} \int_{n, m}^{\prime} \frac{g+x I}{n-2 m} \delta_{n m} \sin \left(n \phi-m \theta+\lambda_{n m}\right) .
$$

The equations are simpler if the force-free current part of $B$ is singled out by defining

$$
\mu=H_{H}+\frac{4 \pi}{B_{0}^{2}} \frac{d P}{d \psi} I
$$

then

$$
B=\mu(\psi)(x \phi-\theta)+\frac{4 \pi}{B_{0}^{2}} \frac{d P}{d \psi}(g \phi+I \theta)+B_{*}
$$

The parallel current 18 given by Eq. (19)

$$
\frac{4 \pi}{c} \frac{1}{\theta}=\mu(\psi)+\frac{4 \pi}{B_{0}^{2}} \frac{d P}{d \psi} \int_{n, m}^{\prime} \frac{n I+m g}{n-m} \delta_{n m} \cos \left(n \phi-m \theta+\lambda_{n m}\right) .
$$

The first term on the right aide of this equation represents the force-free current and the second term the Pfirsch-schlüter current. The poloidal and toroldal furrents can be evaluated using bq. (15) and (25). 


$$
\begin{aligned}
& \frac{d I}{d \psi}=\mu-\left(\frac{4 \pi}{B_{0}^{2}} \frac{d P}{d \psi}\right) I \\
& \frac{d g}{d \psi}=-\psi \mu-\left(\frac{4 \pi}{B_{0}^{2}} \frac{d P}{d \psi}\right) g .
\end{aligned}
$$

Consequently $B$ of Eo. (25) can be rewritten as

$$
\beta=-\frac{d g}{d \psi} \phi-\frac{\sigma I}{d \psi} 9+B_{*}
$$

which identifies the $B_{*}$ of Eq. (23) with that of Eq. (13).

It is of interest to note that in force-free magnetic fields that the plasma is minimum average B stable if B increases away fron the magnetic by the $v^{* *}$ criterion of Johnson and Greene. 7

\section{Consequences of $\mathrm{Ohm}$ 's Law}

The Ohm's Law of magnetohydrodynamics,

$$
t+\frac{1}{C}+\vec{v} \times \vec{B}=\vec{n} \cdot \ddagger
$$

allows us to evaluate a plasma diffusion coefficient. The electric field t is the sum of a potential, - t $\phi$, and a solenoidal, $\vec{\varepsilon}$, part

$$
\vec{E}=-\vec{\nabla} \Phi+\vec{E} \text {. }
$$

The perpendicular part of $\vec{E}$ can be written in terms of a velocity $\vec{u}$ 


$$
\vec{\varepsilon}_{1}+\frac{1}{c} \vec{u} \times \vec{B}=0
$$

The velocity $\vec{u}$ represents an overall pinching of the fleld and the plasma. The ohm's taw can be rewritten as

$$
-\vec{\nabla}_{\phi}+\vec{\varepsilon}_{\|}+\frac{1}{c}(\vec{v}-\vec{u}) \times \vec{B}=\vec{\eta} \cdot \vec{j} .
$$

The parallel component of this equation gives

$$
\frac{\partial \phi}{\partial \phi}+x \frac{\partial \phi}{\partial \theta}=(g+x I)\left(\frac{E_{\Pi}}{B}-\pi_{B} \frac{j}{B}\right)=
$$

This equation with the expression for $j, / B$, Eq * (26), Implies

$$
\begin{aligned}
& \vec{\varepsilon}_{A}=\frac{\pi_{n} c}{4 \pi} \mu(\psi) \stackrel{B}{ } \\
& \Phi=\phi_{0}(\psi)-n_{n} c \frac{(g+x I)}{B_{0}^{2}} \frac{d P}{d \psi} \sum_{n, m} \sum^{\prime} \frac{n I+m g}{(n-1 m)^{2}} \delta_{n m} \sin \left(n \phi-m \theta+\lambda_{n m}\right)
\end{aligned}
$$

with $\phi_{0}$ an arbitrary function of $\psi$.

To understand the velocity $\vec{u}$ we will consider Faraday's Law

$$
\begin{aligned}
& \frac{\partial \vec{B}}{\partial t}=-c \vec{t} \times \vec{E}
\end{aligned}
$$

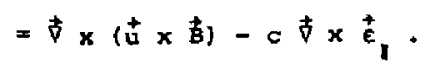

Evaluating $\vec{u} \times$ 血 using the contravariant expression for $\frac{1}{6}$, Bq. (12), one 
finds

$$
\begin{aligned}
& \frac{\partial \psi(\vec{x}, t)}{\partial t}+\dot{u} \cdot \nabla_{\psi}=-\left(-\frac{n c^{2}}{4 \pi} \mu\right) I \\
& \frac{\partial \psi_{p}(x, t)}{\partial t}+\vec{u} \cdot \vec{t} \psi_{p}=\left(\frac{n_{n} c^{2}}{4 r} \mu\right) g
\end{aligned}
$$

with $d \psi_{p} / d \psi=\psi$ one can easily show $2 \pi \psi_{p}$ is the poloidal flux within an additive function of time. One can define the plasm loop voltare $v(\psi, t)$ by

$$
V=\frac{\partial}{\partial \psi}\left(\frac{1}{2 \pi} \quad \int \vec{E} \cdot \vec{B} a^{3} x\right)
$$

in the usual approximations of a tokamak $V \simeq 2 \pi R E_{\phi}$. Evaluating Eq, (40) uging Eq. (35) for $E_{\|}$, one finds

$$
\mu=\frac{2}{c} \frac{v}{(g+x I) n_{\|}}
$$

This equation plus Ers. (38) and (39) imply

$$
\frac{\partial x(\psi, t)}{\partial t}=\frac{c}{2 \pi} \frac{\partial V}{\partial \psi}
$$

In steadystate one must clearly have the loop voltage a constant, $v_{0^{*}}$ one then has

$$
\vec{u} \cdot 7_{\psi}=-\frac{c}{2 \pi} \frac{I}{g+x I} v_{0}
$$

and the total flux of particles due to $\vec{u}$ with $\rho(\psi)$ the density is 
$\|_{1}$

$$
\begin{aligned}
\Gamma_{\mathrm{P}} & =\int \rho \overrightarrow{\mathrm{u}} \cdot \overrightarrow{\mathrm{dS}} \overrightarrow{\mathrm{S}}_{\psi} \\
& =-2 \pi \in \frac{\rho I}{\mathrm{~B}^{2}} \mathrm{v}_{0} .
\end{aligned}
$$

This is the classical pinch effect.

The particle diffusive flux can be evaluated using chm's Law, Eq. (33). This equation can be solved for the perpendicilar part of + and hence $(\vec{v}-\vec{t}\} \cdot \vec{\nabla} \psi$. One finds, using Eq. $(36)$,

$$
\begin{aligned}
& (\vec{v}-\vec{u}) \cdot \vec{\nabla}_{\psi}=\frac{c}{g+\alpha I}\left(I \frac{\partial \phi}{\partial \phi}-g \frac{\partial \phi}{\partial \theta}\right)-\eta_{1} c^{2} \frac{\vec{\nabla} P}{B^{2}} \cdot \vec{\nabla} \psi \\
& =-\frac{n_{1} c^{2}}{B_{0}^{2}} \frac{d P}{d \psi} \int_{n, m}^{\prime}\left(\frac{\pi I+m g}{n-x m}\right)^{2} \delta_{n m} \cos \left(n \phi-m \theta+\lambda_{n m}\right)-n_{1} c^{2} \frac{d P}{d \psi} \frac{|\nabla \psi|^{2}}{B^{2}} \text {. }
\end{aligned}
$$

The total diffusive particle flux crossing a magnetic surface is

$$
\begin{aligned}
& r_{D}=\int \rho(\vec{v}-\vec{u}) \cdot \frac{\vec{\nabla} \psi}{\vec{\nabla}_{\phi} \cdot(\vec{\nabla} \psi x \vec{\nabla} \theta)} \mathrm{d} \theta \mathrm{d} \phi \\
& =(g+x I) \rho \int(\vec{v}-\stackrel{+}{\mathbf{v}}) \cdot \frac{\vec{\nabla}}{\psi} \frac{\mathrm{d} \theta \mathrm{d} \phi}{\mathrm{B}^{2}} .
\end{aligned}
$$

The expression for the total diffusive flux can be rewritten as

$$
r_{D}=-\left(D_{11}+D_{1}\right) \frac{d P}{d \psi}
$$

with 


$$
\begin{aligned}
& D_{\|}=2 \pi^{2} n_{\|} \frac{c^{2}(g++I)}{B_{0}^{4}} \sum_{n, m}^{\prime}\left(\frac{n I+m g}{n-x m}\right)^{2} \delta_{n m}^{2} \\
& D_{\perp}=n_{\perp} c^{2} \int \frac{\nabla_{\psi}}{B^{2}} \cdot d \vec{s}_{\psi}, d s_{\psi}=\frac{\nabla_{\psi}}{\nabla_{\phi} \cdot\left(\vec{\nabla}_{\psi} x^{+} \theta\right)} d \theta d \phi,
\end{aligned}
$$

that is, $d \xi_{\psi}$ is the area element of a flux surface.

Let us assume the plasma is in a steady state. Then particle conservation implies

$$
\dot{f} \cdot \mathrm{n} \mathfrak{v}=\mathrm{g}
$$

with $s$ the source of particles per unit volume. The total flux of particles $\Gamma=\Gamma_{p}+\Gamma_{D}$ obeys

$$
\begin{aligned}
\frac{d \Gamma}{d \psi} & =\int s \frac{d \theta d \phi}{\vec{\nabla} \phi \cdot(\vec{\nabla} \psi \times \vec{\nabla} \theta)} \\
& =s(\psi)
\end{aligned}
$$

with $s(\psi)$ d $\psi$ the number of particles added between two differentially separated flux surfaces. Clearly I must be a smoothly varying function of $\psi$.

\section{v. Conclusions}

In steadystate the total flux of particles across a magnetic aurface is

$$
\Gamma=-D(\psi) \frac{d P}{d \psi}+\Gamma_{P}
$$


with $D(\psi)=D_{n}+D_{1}$ given by Eqg. $(48)$ and (49) and $T_{p}$ given by Eq. (44). The parallel current driven aifferention coefficienc $D_{y}$ ts singular at each rational surface. That is, near a ratsonal surface

$$
D_{n} \propto \frac{\delta_{n m}{ }^{2}}{\left(\psi-\psi_{n m}\right)^{2}}
$$

with $\psi_{n m}$ the value of $\psi$ when $n=2 m$. The total particle flux $T(\psi)$ must be slowly varying in $\psi$ so we find near a rational surfare

$$
\frac{d p}{d \psi} \propto\left(\psi-\psi_{n m}\right)^{2} / \delta_{n m}^{2}
$$

Conseguently, unless $1 \delta_{\mathrm{nm}} \mid=0$ at $\psi=\psi_{\mathrm{nm}}, \mathrm{dp} / \mathrm{d} \psi$ vanishes at rational surfaces. The Pfirsch-sehlüter or pressure driven part of parall 1 current near a rational surface [see $(26)$ ] is

$$
\begin{aligned}
& \left(j_{P}\right)_{P} \times \frac{\delta_{n m}}{\psi-\psi_{m m}} \frac{d P}{d \psi} \cos (n \phi-m \theta) \\
& \Leftrightarrow\left(\psi-\psi_{n m}\right) .
\end{aligned}
$$

Congequently the PEIrach-5chlüter part of $j$, vanishes at each rational surface rather than being sirgular and the pregaure driven part of $j$ goes to zero everywhere as the plasma pressure goes to zero. The electrostatic potential, Intereding1y, retains a finite varkation on rational magnetic surfaces even though dP/dip $1 \mathrm{~s}$ zero on these vurfaces.

The gingularity of $D(\psi)$ at ebch rational surface is not as important as 1t might first appesr. Considex a region of the plasma with no sources

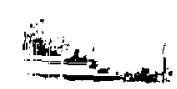


so $d \Gamma / d \psi=0$. Noifine a smoothing function $\Delta(\psi)$ auch that $\Delta(\psi)$ goes to zero for $|\psi|$ smali but Einite and whtch has a unit tntegral over $\psi \cdot$ Tiner: let

$$
\begin{aligned}
\overline{\mathbf{P}}(\psi) & \equiv \int \Delta\left(\psi-\psi_{1}\right) P\left(\psi_{1}\right) \mathrm{d} \psi_{1} \\
\frac{\mathrm{d} \overline{\mathrm{P}}}{\mathrm{d} \psi} & =-\int \frac{\mathrm{d} \Delta}{\mathrm{d} \psi_{1}} P\left(\psi_{1}\right) \mathrm{d} \psi_{1} \\
& =+\int \Delta \frac{\mathrm{dP}}{\mathrm{d} \psi_{1}} \mathrm{~d} \psi_{1} \\
& =-\Gamma \int \frac{\Delta\left(\psi-\psi_{1}\right)}{\Omega\left(\psi_{1}\right)} \mathrm{d} \psi_{1} .
\end{aligned}
$$

Defining

$$
\frac{1}{\bar{D}(\psi)} \equiv \int \frac{\Delta\left(\psi-\psi_{1}\right)}{D\left(\psi_{1}\right)} \mathrm{d} \psi_{1} \text {. }
$$

one has

$$
\Gamma=-\bar{D}(\psi) \frac{d \bar{p}}{d \psi}
$$

No matter how narrow the region over which $\Delta$ is different from zero, as long as the region is finite, the function $\bar{D}(\psi)$ is finite everywhere. Thts follows from the fact that the Fourier transform of a smooth function vanishes exponentially for high $n$ or $m$; hence high order rational surfaces have a exponentially gmall effect on the integral leading to $\bar{D}$.

An Interesting applicstion of the expression for $D_{1}$ is to derive the well-known Pfirsch-Gchlüter aiffusion coefficient for a stellarator. This is dirived by assuming the field strength has the obvious form 


$$
\frac{1}{B^{2}} \simeq \frac{1}{B_{o}^{2}}\left[1-2 E \cos \theta-2 \varepsilon \cos \left(\mathrm{N}_{\phi}-\ell \theta\right)\right] .
$$

The only terms to $\delta_{\mathrm{nm}}$ are $\delta_{\mathrm{O} 1}=2 \mathrm{E}$ and $\delta_{\mathrm{NQ}}=2 \delta$. We assume the plasma hae negligible net toroidal current, $I=0$, and the toroidal field dominates go $g=R B_{0}$. The diffusion coeffictent one la used to seeilg $D_{\|}^{*}$ is $D_{\|}$divided by the area of the magnetic surface $(2 \pi r)(2 \pi R)$ and also divided by $d \psi / d r=r B_{0}$ since the usual $D_{n}^{\star}$ muliiplies $d p / d r$ rather than $d P / d \psi$. Eq. (48) implies witr. $\varepsilon=r / R$

$$
D_{1}^{*}=\frac{2}{x^{2}} \frac{n_{n} c^{2}}{B_{0}^{2}} \sum_{n, m}^{-}\left(\frac{\delta_{n m}}{2 \varepsilon}\right)^{2}\left(\frac{x}{x-\frac{n}{m}}\right)^{2} .
$$

The resonance $\mathrm{n}=0, \mathrm{~m}=1$, with $\delta_{01}=2 \mathrm{E}$ gives a contribution

$$
D_{01}^{*}=\frac{2}{x^{2}} \frac{n_{n} c^{2}}{B_{0}^{2}}
$$

the Pfirsch-Sch+üter coefflcient. The resonance at $n=N, m=\ell$,

$$
\begin{aligned}
& \delta_{\mathrm{N} R}=2 \delta \text { gives } \\
& \mathrm{D}_{\mathrm{N} \ell}^{\star}=\frac{2}{x^{2}} \frac{n_{n} c^{2}}{\mathrm{~B}_{\mathrm{O}}^{2}}\left(\frac{\delta}{\mathrm{E}}\right)^{2}\left(-\frac{l}{z-\mathrm{N} / \mathrm{l}}\right)^{2} .
\end{aligned}
$$

Customary stellarator designg have $E \sim E$ but $N / \ell s$ so the pfirgch-Schlüter coefficlent gives an accurate approximation, $\mathrm{D}_{01}>\mathrm{D}^{*} \mathrm{~N}$. 
$-17-$

Acknowledgnests

Th1s work was oupported by U.S. Department of Energy contract no. UEAC02-76-CH03073. 
References

1. H. Grad, Phys. Fluids 10, 137 (1967) (see esp. Sec. 4).

2. M. D. Krugkal and R. M. Kularud, Fhys. Fluids 1, 265 (1958).

3. S. Hanada, Nucl. Fusion 2 , 23 (1962).

4. J. M. Greene and J. L. Johnson, Phys. Fluids 5, 510 (1962).

5. H. Grad, Courant Institute of Math. Sc. Rept. C00-3077-164, Dec. 1979.

6. A. H. Boozer, Phys. Fluids 22, 904 (1980).

7. J. L. Johnson and J. M. Greene, Plasma Physics 9, 611 (1967). 